\title{
Laparoscopic Staple-less Splenectomy: feasibility and benefits
}

\author{
Hesham Ahmed Nafady ${ }^{1}$, Ahmed Mohamed Rashad ${ }^{1}$, Mohammed Nady Sayed ${ }^{1}$ \\ ${ }^{1}$ General Surgery Department, Faculty of Medicine, Beni-Suef University
}

\section{ABSTRACT}

Background: The surgical procedures of the spleen include open splenectomy (OS) laparoscopic splenectomy (LS). Several techniques for control of splenic vessels during LS have been developed, such as Clips, sutures, bipolar vessel sealing devices as Liga-Sure and ultrasonic devices as Harmonic have. The vessel sealing devices shows some advantages if compared with other methods such as staplers and clips as shorter hospital stay, safer, feasible and excellent cosmetic results. And vessel sealing devices (Liga-Sure) LS is cheap alternative to the use of endo-staplers which is expensive. Objectives: The study is to evaluate feasibility, merits and demerits of staple-less laparoscopic splenectomy in cases of non-traumatic disorders of spleen and assess operative time, need for blood transfusion, rate of conversion to open splenectomy or use stapler, period of hospital stay and associated complications. Patients and Methods: The study was conducted on 32 patients, underwent laparoscopic splenectomy using vessel sealing devices (Ligasure) for splenic pedicle control. Informed consent was obtained for all patients. Results: The study was conducted on 32 patients. 30 patients $(93.80 \%)$ were females and 2 patients $(6.3 \%)$ were males. 30 patient with immune thrombocytopenic purpura (ITP) and 2 patients with hereditary spherocytosis (HS). Two cases (6.3\%) detected with accessory spleen (splenule) at the splenic hilum. Two cases $(6.3 \%)$ were converted to open splenectomy. To control bleeding, we used stapler in two cases (6.3\%). Cost of staple-less LS is less expensive than stapler laparoscopic splenectomy as the stapler LS is more than staple-less LS by about 12000 EGP (700\$). Conclusion: In a report in early 1990s, the dissection of the hilum of the spleen was generally achieved by surgical clips or endo-gastrointestinal staplers. Subsequently, new surgical tools such as ultrasonic devices or bipolar electrosurgical devices such as LigaSure had been employed as supporting device that can seal blood vessels with supra-physiologic burst pressures equal to those obtained with surgical clips or ligatures. We, in this series, believe that it is, with a statistical significance, safe and effective, reduces blood loss, minimize operating time, fewer costs and is a valid. Moreover, it reduced conversion rates in selected patients. Last but not least, the use of the vessel sealing devices (Ligasure) resulted in no post-operative complications or need of blood transfusion.

Keywords: Staple-less, Laparoscopic Splenectomy, Ligasure, Vessel sealing device. 


\section{Introduction}

The spleen is the largest organ of the lymphoreticular system. Its primary functions include hematopoiesis and immune protection. It acts as a filter for abnormally shaped blood cells and some metabolic products. Also, it has the ability to store blood and contract in response to acute changes in blood volume in trauma and it becomes the focus of surgical attention in a variety of circumstances. The spleen contains phagocytes that remove exhausted erythrocytes and foreign bodies from the blood and it is a major reservoir for blood. Absence of the spleen predisposes to certain infections (1).

Indications of splenectomy include hemolytic disorders like ITP (Idiopathic thrombocytopenic purpura), Thalassemia, Idiopathic autoimmune hemolytic anemia, sickle cell anemia, traumatic rupture spleen, secondary hypersplenism, splenic cysts, Elliptocytosis, Spherocytosis, splenic artery aneurysm and splenic vein thrombosis (2).

The surgical procedures of the spleen include total open splenectomy (OS) (The first splenectomy done by Zacarello in 1549), partial splenectomy, laparoscopic splenectomy (LS) (In 1991, Delaitre reported the first laparoscopic splenectomy), splenic repair and splenic fixation. Laparoscopic splenectomy is a gold standard procedure that can be done by anterior, semi lateral, or lateral approach. In comparison to the open technique, the laparoscopic method is safer, effective, less postoperative pain; rapid recovery and shorter hospital stay (3).
Several techniques for control of splenic vessels during laparoscopic Splenectomy have been developed, such as Clips, sutures, monopolar, bipolar vessel sealing devices as Liga-Sure and ultrasonic devices as Harmonic have used as haemostatic methods. The vessel sealing devices shows some advantages if compared with other methods such as staplers and clips; easier dissection of spleen so the risk of injury to pancreatic tail or stomach is decreased, less expensive, minimal lateral thermal spread $2 \mathrm{~mm}$, frequent instrument interchange during using other methods is avoided as grasping, coagulation and cutting are done by the same device saving time and decreasing possibility of any organ injury. Also, no foreign bodies are left after hemostasis such as clips and sutures (4).

\section{Aim of the work}

To evaluate feasibility, merits and demerits of staple-less laparoscopic splenectomy in cases of non-traumatic disorders of spleen.

\section{Patients and Methods}

The study was conducted at the surgical department of Beni-Suef University and Kobry El-kobba hospitals, within the period from August 2018 to August 2019. Most cases were referred by hematology department as candidates for splenectomy due to hematological diseases. The study was conducted on 32 patients, underwent laparoscopic splenectomy using vessel sealing device (Ligasure) for splenic pedicle control. Informed consent was obtained for all patients, to assess operative time, cost, need for blood 
transfusion, rate of conversion to open splenectomy or use stapler, time of hospital stay.

\section{Inclusion criteria}

1 - Any cases with splenomegaly $(<20 \mathrm{~cm})$ with an indication for splenectomy (e.g. hematological causes: ITP, Spherocytosis).

2- Splenic artery less than $7 \mathrm{~mm}$ in diameter as measured by Duplex.

\section{Exclusion Criteria}

1. Coagulopathy.

2. Cardiopulmonary contraindications of laparoscopic surgery.

\section{Method of the study}

\section{Preoperative work up}

1. Detailed history taking.

2. General examination.

3. Local examination.

4. Investigations (lab. and imaging)

Laboratory studies: full labs as:

- Full pre-operative labs including Coagulation profile: prothrombin time, INR, bleeding and clotting time.

\section{Imaging studies:}

- Abdominal US was done for all patients with full comment on the following:

- Spleens as regard its size, span, presence or absence of any focal lesion, splenic vessels diameter by (duplex).

- Ascites, gall bladder stones or cholecystitis.

\section{Preoperative preparation:}

For patients presented with blood picture alteration as reduced hemoglobin percentage and low platelet count.
- Packed red blood cells transfusion for those with low hemoglobin percentage aiming to raise its level above $10 \mathrm{gm} \%$.

- For patients with low platelet count as ITP and those with autoimmune hemolytic anemia a 30-40 mg of Prednisolone was given twice daily and high dose of immunoglobulin G(400 $\mathrm{mg} / \mathrm{kg}$ per day) 3-5 days prior to surgery aiming to raise the platelet count up to 70,000 $/ \mathrm{dl}$.

- Vaccinations with Pneumococcal PPV 23 vaccine, Meningococcal $\mathrm{C}$ vaccine and $\mathrm{H}$. influenza $b$ vaccine 2 weeks pre-operatively.

- Antibiotic prophylaxis with preoperative single dose ampicillin sulpactam $1.5 \mathrm{~g}$ vial IV.

- Preoperative preparation of packed RBCs units and FFP units for unexpected intra-operative hemorrhage.

- Fasting period ranged from 6 to 8 hours.

\section{Our technique procedure:}

Here the patient lies in a supine decubitus and the table is angulated at the level of patient umbilicus to gain a space between the costal margin and iliac crest. Using five trocars, the first one is $10-\mathrm{mm}$ trocar about $4 \mathrm{~cm}$ below costal margin in mid-clavicular line in the lateral border of rectus sheath for 10-mm 30 degree telescope and inflation is done from this trocar, A pneumoperitoneum started up to pressure of 15 $\mathrm{mm} \mathrm{Hg}$. this site could be moved upwards or down wards according to splenic size, the second trocar $10-\mathrm{mm}$ is applied hand breadth $5 \mathrm{~cm}$ to the left side of telescope port (anterior axillary line ) for the surgeon's right hand for application of the 
vessel sealing devices (Ligasure), the third trocar is $5-\mathrm{mm}$ one applied at $5-7 \mathrm{~cm}$ to right side of telescope port for the surgeon's left hand. The fourth trocar $10-\mathrm{mm}$ is placed just below xiphisternum for the retractor of the liver. The fifth trocar 5-mm is placed in left mid-axillary line midway between left costal margin and left iliac crest used by the assistant who could be omitted in cases with small spleen.

First insert a liver retractor to keep the left lobe of the liver out of the operative field from the third trocar. The stomach is retracted medially and the spleen is exposed. Starting with gastrosplenic ligament, the dissection continues using vessel sealing devices (Ligasure).

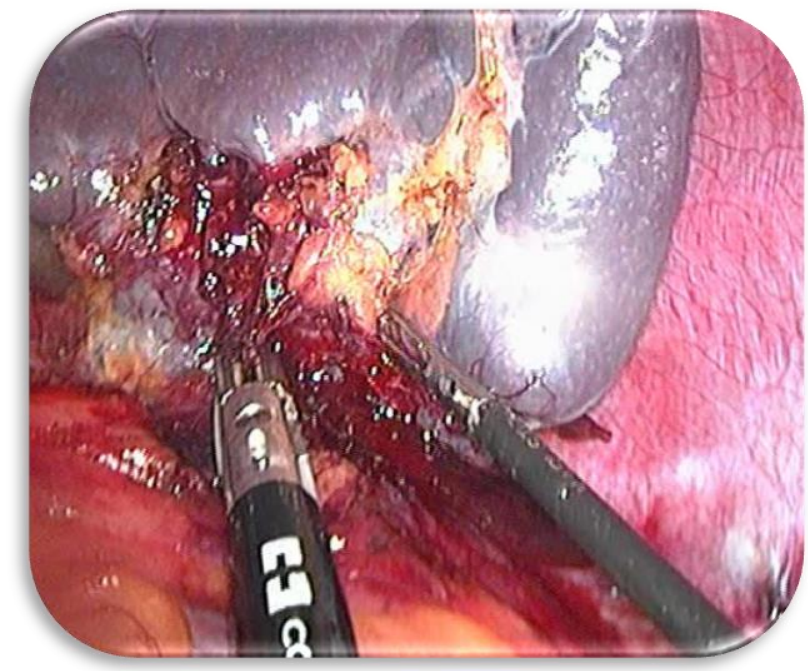

Figure (1): Showing Control of the splenic pedicle using Ligasure.

Putting a drain and the dissection area reinspected to ensure hemostasis, and to exclude injures to the stomach, pancreas, diaphragm, and colon. Finally, delivery of spleen through a bag or with a Pfannenstiel incision. Then closure of incisions in layers. medially and superiorly, so that the short gastric vessels are divided. Short gastric vessels were controlled after opening the lesser sac using vessel sealing devices (Ligasure) with care of good visualization of the splenic pedicle. Then we divide spleno-colic ligament from the colon splenic flexure which moves away from the spleen by its weight. Then we divide the splenorenal ligament from downward. Then the superior pole of the spleen is completely mobilized by taking down the uppermost portion of the splenorenal ligament. The complete mobilization of the superior pole of the spleen will subsequently facilitate the vascular control of the splenic hilum

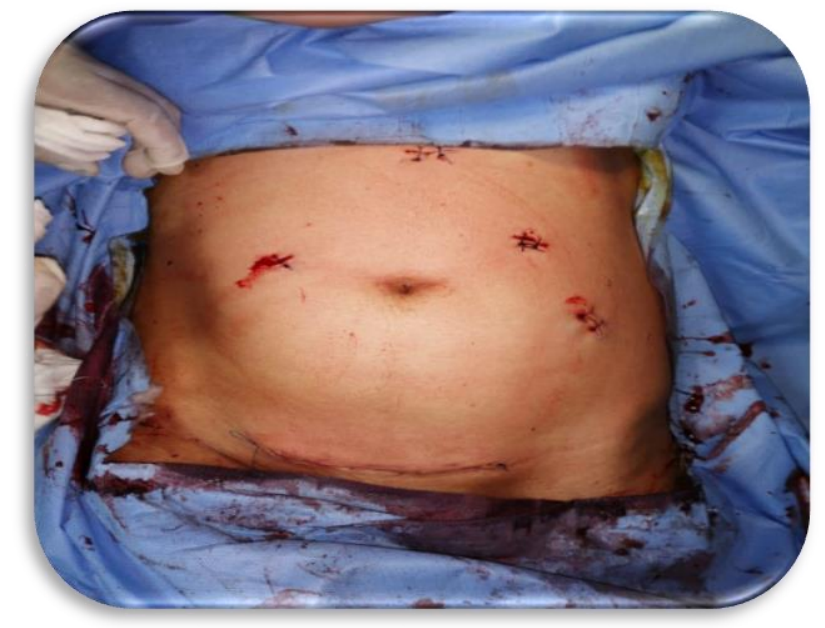

Figure (2): Postoperative picture with a Pfannenstiel incision after closure on layers.

\section{Postoperative care and follow up:}

All patients were hospitalized postoperative with daily assessment by complete blood count to monitor the platelets count, hemoglobin level and white blood cell count. The drain removed only when non bloody fluid less than $30 \mathrm{ml}$ in 24 hours. The patient started oral intake once good intestinal sounds could be auscultated. Follow up abdominal US for excluding any collections. The 
patients were followed in the outpatient clinic by local examination, complete blood count to assess the platelets count and degree of reactive thrombosis thrombocytosis for fear of post-operative complications as portal vein thrombosis (PVT), pulmonary embolism (PE) and deep vein

(DVT).

\section{Results}

Table (1): Sex of groups

\begin{tabular}{|c|c|c|c|}
\hline \multicolumn{2}{|c|}{} & Frequency & Percent \\
\hline \multirow{3}{*}{ Valid } & Female & 30 & 93.8 \\
\cline { 2 - 4 } & Male & 2 & 6.3 \\
\cline { 2 - 4 } & Total & 32 & 100.0 \\
\hline
\end{tabular}

30 patients (93.80\%) were females and 2 patients (6.3\%) were males.

Table (2): Disease

\begin{tabular}{|c|c|c|c|}
\hline \multicolumn{2}{|c|}{} & Frequency & Percent \\
\hline \multirow{3}{*}{ Valid } & ITP & 30 & 93.8 \\
\cline { 2 - 4 } & Spherocytosis & 2 & 6.3 \\
\cline { 2 - 4 } & Total & 32 & 100.0 \\
\hline
\end{tabular}

Table (3): Other findings

\begin{tabular}{|c|c|c|c|}
\hline \multicolumn{2}{|c|}{} & Frequency & Percent \\
\hline \multirow{4}{*}{ Valid } & No other findings & 28 & 87.5 \\
\cline { 2 - 4 } & Splenule & 2 & 6.3 \\
\cline { 2 - 4 } & HTN & 2 & 6.3 \\
\cline { 2 - 4 } & Total & 32 & 100.0 \\
\hline
\end{tabular}

Table (4): Conversion to open

\begin{tabular}{|c|c|c|c|}
\hline \multicolumn{2}{|c|}{} & Frequency & Percent \\
\hline \multirow{3}{*}{ Valid } & no & 30 & 93.8 \\
\cline { 2 - 4 } & yes & 2 & 6.3 \\
\cline { 2 - 4 } & Total & 32 & 100.0 \\
\hline
\end{tabular}

Two cases $(6.3 \%)$ were converted intra-operatively to open splenectomy due to bleeding from splenic artery trunk or vein at splenic hilum with failure of control of bleeding vessel due to large splenic artery size (one case was $6.2 \mathrm{~mm}$ and the other was $7 \mathrm{~mm}$ ) and big spleen $20 \mathrm{~cm}$.

Table (5): Use of stapler

\begin{tabular}{|c|c|c|c|}
\hline \multicolumn{2}{|c|}{} & Frequency & Percent \\
\hline \multirow{3}{*}{ Valid } & no & 30 & 93.8 \\
\cline { 2 - 4 } & yes & 2 & 6.3 \\
\cline { 2 - 4 } & Total & 32 & 100.0 \\
\hline
\end{tabular}

To control bleeding, we used stapler in two cases (6.3\%). 
Table (6): Descriptive Statistics

\begin{tabular}{|l|c|c|c|c|c|}
\hline & $\mathbf{N}$ & Minimum & Maximum & Mean & Std. Deviation \\
\hline age of study group & 32 & 18 & 50 & 28.69 & 10.524 \\
\hline Size of spleen & 32 & 12 & 20 & 14.51 & 1.850 \\
\hline Diameter of splenic artery & 32 & 5 & 7 & 5.61 & .436 \\
\hline WBCs & 32 & 5.2 & 15.1 & 8.394 & 2.6378 \\
\hline $\mathrm{Hb}$ & 32 & 10.0 & 15.5 & 12.888 & 1.5722 \\
\hline Platelet count & 32 & 65 & 300 & 111.81 & 57.951 \\
\hline Hospital stay & 32 & 2 & 5 & 3.56 & .814 \\
\hline Blood loss & 32 & 100 & 500 & 252.50 & 113.695 \\
\hline Operative time & 32 & 60 & 120 & 80.62 & 14.818 \\
\hline Valid N (listwise) & 32 & & & & \\
\hline
\end{tabular}

- Cost of staple-less laparoscopic splenectomy is less expensive than stapler laparoscopic splenectomy as the stapler cost 6000 EGP about (350\$) and two cartridge at least are used they cost also about 6000EGP (350\$) so that the stapler laparoscopic splenectomy is more than staple-less laparoscopic splenectomy by about 12000 EGP (700\$).

- There is no postoperative bleeding detected as a complication in our study.

\section{Discussion}

Laparoscopic splenectomy (LS) has evolved over the past 15 to 20 years to become the preferred approach for splenectomy. Less pain, shorter hospitalization, faster return to regular activities, and smaller scars are the main advantages over open splenectomy (5). In 1991, Delaitre reported the first laparoscopic splenectomy. Since then laparoscopic splenectomy has become the procedure of choice to treat hematological diseases requiring splenectomy (3).

Knowledge of anatomical details and appropriate surgical skills are the sine-qua-non conditions to reduce, significantly the incidence of these complications (6).

In a report in early 1990s, the dissection of the hilum of the spleen was generally achieved by surgical clips or endo- gastrointestinal staplers. Subsequently, new surgical tools such as ultrasonic devices or bipolar electrosurgical devices such as LigaSure have been employed as supporting devices that can seal blood vessels with supraphysiologic burst pressures equal to those obtained with surgical clips or ligatures (7). Several reports indicate that LigaSure has been shown to withstand a minimum of 3 times the normal systolic pressure, and they have been approved by the Food and Drug Administration for sealing vessels up to a vessel diameter $7 \mathrm{~mm}$. Several reports support sutureless and stapleless LS using LigaSure as a safe and timesparing procedure (8).

Huge splenomegaly was considered as contraindication for LS in our study and patients were excluded from the study. Gianpaolo and 
collegues included such patients in their study and concluded that no specific condition can be considered as an absolute contraindication to LS in experienced centers, except for massive splenomegaly (9).

Blood loss during laparoscopy was one of the assessed variables in the present study and it revealed mean blood loss of $252.50 \pm 111 \mathrm{ml}$, while in the study by Ji and Liu the mean volume of blood loss was $500 \pm 94 \mathrm{~mL}(\mathbf{1 0})$ and $80 \mathrm{~mL}$ in (11) and 198.5 in (12) and was $131.7 \mathrm{~mL}$ in (13) and The mean estimated loss of bleeding was $245 \mathrm{ml}$ in (14).

One of main concerns about LS is its ability to search for and detect accessory spleen at its expected sites. In our study, 2 cases were associated with accessory spleen with rate of (6.3\%). Accessory spleens were recognized and removed in eight patients (12.6\%) (8). In three patients (15\%), accessory spleens were detected during operation, which were removed (13).

Intraoperative bleeding is the major concern with LS due to the complex splenic blood supply and the difficultly of dissection associated with the frailty of the parenchyma. The high blood loss sometimes reported in the literature is harmful for the patient and makes the dissection difficult due to inadequate visualization of anatomical structures. Furthermore, reported conversion rates are usually between 5 and 10\% (8).

In our study, Intraoperative complications were revealed in 4 out of 32 patients, they had intraoperative bleeding most probably due to the low preoperative platelet count, big splenic artery and vein diameters or big spleen. We used stapler in two cases and two cases were converted to open splenectomy intra-operatively due to bleeding from splenic artery trunk or vein at splenic hilum and were in relatively large spleen. Conversion rate to open is $6.3 \%$. In the study by Romano and Gelmini reported Conversion was necessary in five cases of 63 cases $(7.9 \%)$ due to hilar bleeding in three cases (two due to incorrect use of (LigaSure), difficult splenic hilar dissection in one case, and massive splenomegaly in one case. No other cases of intraoperative bleeding due to LigaSure were recorded, additional clipping was never necessary, but in only one case using of an EndoGIA stapler for the splenic artery because of its large size (8). Ji and lie reported Conversion to open was necessary in two cases out of 105 cases (1.9\%), 103 patients (98.1\%) underwent successful LS (10). Romano and Gelmini reported Conversion was necessary in 9 cases $(8.1 \%)$, owing to hilar bleeding in 6 cases, capsular bleeding in 1 case, difficult splenic hilar dissection in 1 case, and massive splenomegaly in the latest case (12). No conversion to open procedure in the study of (13). Conversion rate was $(9.8 \%)$ of 51 patients in study of (14). Xiao and colleagues, in their study on LS in massive splenomegaly, reported conversion rate $13.3 \%$ (4 converted cases in overall 34 LS case) (15). The difference in conversion rate between these two studies 
reflects the effect of size of spleen and diameter of splenic vessels as challenging factors in LS.

In our study no postoperative bleeding reported, no case had late thrombotic events, no case had sub-phrenic abscess no case developed pneumonia, no chest infection, fever and /or ileus. Nada et al. reported that There was no perioperative mortality, with three $(12.5 \%)$ postoperative complications, 2 cases with thrombosis of the spleno-portal axis treated conservatively and one case with postoperative fever due to intraabdominal hematoma drained under ultra-sonographic guidance (16). Postoperative complications occurred in 1 patient with bowel obstruction symptoms that underwent surgery (13). There was no postoperative mortality, with a major complication rate of $8.1 \%$ ( 9 cases), owing to a case of spleno-portal vein thrombosis discovered 10 days after LS, which required a hospital readmission and a heparin infusion, a hemo-peritoneum owing to a bleeding of the pancreatic tail (2 cases) and port-site hemorrhage ( 2 cases) both laparoscopically treated superficial surgical wound infection (1 case) and pleural effusion that was medically treated (3 cases) (12). Patients were followed up for 3 months to 2 years, showing no occurrence of major complications (10). There were no postoperative complications (17).

Our study reported shorter mean hospital stay 3.56days with range (2-5 days), compared to Amau et al. (18) who declared mean hospital stay 3.8 days in a stapler laparoscopic splenectomy. Vecchio and colleagues reported Mean hospital stay of 3.5 days with range (range 3 - 7 days) (2). Zhu and collegues reported mean post-operative hospital stay 3.5 days in LS in comparison to 5.7 days in OS (19). Hassan and Al Ali reported mean postoperative hospital stay 1.5 days in LS in comparison to 4 days in OS (20). Like any other laparoscopic operations, LS is associated with rabid recovery and early rehabilitation. So, shortening post-operative hospital stay. Romano et al., The average postoperative stay was 4.2 days (range, 3-7) (8). Ji and Liu reported that the mean duration of hospital stay was 7.5 days (range: 6-9 d) (10), and the mean is 5.2 days in (14).

One of reported items in LS is operative time. In agreements with our data several authors have demonstrated a significant decrease in LS surgical time with gaining experience in LS. In this study, Mean operative time \pm SD is $80.62 \pm 14.8$ min. Hassan and $\mathrm{Al}$ Ali reported mean operative time $180 \mathrm{~m}$ in LS Vs $120 \mathrm{~m}$ in OS (20). Fujioka reported that the operative times were 90 minutes (17). Romano and Gelmini reported that the average operating time was 120min (range, 65-250) (8). Ji and liu reported the mean operative time was $100 \mathrm{~min}$ (10). Romano reported that mean operative time was 120min (range 90-165) (11). The mean operating time is $141 \mathrm{~min}$ in (14) and Shabahang reported that operating time was $115.4 \mathrm{~min}$ in the clip group and $100.6 \mathrm{~min}$ in the ligasure group (13). 
Generally, prolonged operative time in LS may be attributed to skills and experience of the operator and moreover, there is consumed time in extracting the spleen through endo-bag or through mini laparotomy. Hassan and Al Ali study reflected the effect of hugely enlarged spleen on prolongation of operative time in LS in comparison to the same cases in OS.

Splenic pedicle control is the most important step in LS. We utilized vessel sealing devices (Liga-Sure) to control the pedicle as it is available in our hospital, needs less dissection and shorten operative time. As gelmini and romano utilized the same maneuver (8), Xiao and collegues controlled the pedicle using $10 \mathrm{~mm}$. titanium clips and vessels sealing with ligature (15). Splenic retrieval was done by endobag morcellation unless the spleen was required for histopathology or it was large for the endo-bag where it was removed by Pfannenstiel incision at the end of the procedures. Drain was left in splenic bed to be removed when daily output is less than $50 \mathrm{cc}$ (16).

The dissection of the spleen was completed and the specimen was removed by morselation within a retrieval bag (Endo-pouch, Ethicon, Somerville, NJ, USA) in 51 patients or via a 5or $6-\mathrm{cm}$ incision in 12 patients with a very large spleen or because the pathologist required an entire specimen (8). The dissection of the spleen is completed and the specimen is removed by morselation within a retrieval bag (Endopouch, Ethicon Inc, Somerville, NJ, USA) in 8 cases or via a 4 to $5-\mathrm{cm}$ left subcostal incision in 2 cases in which the spleen was too large (diameter of 22 and $25 \mathrm{~cm})(\mathbf{1 1})$.

In our study none of our patients required blood transfusion like the study of $(\mathbf{8}, \mathbf{1 0}, 13$ and 16).

\section{Conclusion}

In general LS in comparison to OS was shorter hospital stay, safer, feasible, quicker return to everyday activities and excellent cosmetic results. And vessel sealing devices (Liga-Sure) LS is cheap alternative to the use of endo-staplers which is expensive.

\section{References}

1. Puri P, Höllwarth ME. Pediatric surgery: diagnosis and management. Springer Science \& Business Media, 2009.

2. Vecchio R, Intagliata E, Marchese S, La Corte F, Cacciola RR, Cacciola E. Laparoscopic splenectomy coupled with laparoscopic cholecystectomy. Journal of the Society of Laparoendoscopic Surgeons, 2014; 18(2): 252-257.

3. Alwabari A, Parida L, Al-Salem AH. Laparoscopic Splenectomy for Children with Hematological Disorders: Our Initial Experience with 45 Cases, JPSS. 2014; 8: 152.

4. Katkhouda N, Mavor E. Laparoscopic splenectomy. Surg Clin North Am. 2000; 80: 1285-1297.

5. Holcomb GW, Murphy JP. Ashcraft's pediatric surgery, 2010;47: 616-619. 
6. Rodeghiero F, Ruggeri M. Short- and longterm risks of splenectomy for benign haematological disorders: should we revisit the indications? $\mathrm{Br} \mathrm{J}$ Haematol. 2012; 158(1):16-29.

7. Harold KL, Schlinkert RT, Mann DK, Reeder CB, Noel P, Fitch TR, Braich TA, Camoriano JK. (2003): Long-term results of laparoscopic splenectomy for immune thrombocytopenic purpura. Mayo Clin Proc. 2003; 74: 37-39.

8. Romano F, Gelmini R, Caprotti R, Quaranta N, Tazzioli G, Colombo G, Saviano M, Uggeri F. Sutureless and staple-less laparoscopic splenectomy using Radiofrequency LigaSure device. Surg Endosc. 2006; 20: 991-994.

9. Gianpaolo M, Vincenzo S, Aldo R, Giuseppe S, Carla M, Antonio C. Laparoscopic splenectomy: a single center experience. Unusual cases and expanded inclusion criteria for laparoscopic approach. Updates Surg. 2013; 65:115-119.

10. Ji B, Liu Y, Zhang P, Wang Y, Wang G. (2012): A two-step control of secondary splenic pedicles using ligasure during laparoscopic splenectomy. International journal of medical sciences, 2012; 9(9):743747.

11. Romano F, Franciosi C, Caprotti R, De Fina S, Colombo G, Uggeri F. Laparoscopic splenectomy using ligasure. Surg Endosc. 2002; 16: 1608-1611.

12. Romano F, Gelmini R, Caprotti R. Laparoscopic splenectomy: Ligasure versus
EndoGIA: A comparative study. J Laparoendosc Adv Surg Tech. 2007; 17:763-7.

13. Shabahang H, Maddah G, Tavassoli A, Jangjoo A, Alvandipour M, Abdollahi A, Noorshafiee S. Laparoscopic Splenectomy: Ligasure or Clip Ligation?. Surg Laparosc Endosc Percutan Tech. 2012; 22:136-138.

14. Tan M, Zheng CX, Wu ZM, Chen GT, Zhao ZX. Laparoscopic splenectomy: the latest technical evaluation. World J Gastroenterol. 2013; 9:1086-1089.

15. Xiao DJ, Jakimowicz JJ, Albayrak A, Goossens RH. Ergonomic factors on task performance in laparoscopic surgery training. Applied ergonomics. 2012; 43(3): 548-53.

16. Nada AA, El Samadoni A, Mohsen A. Staple-less Laparoscopic Splenectomy; Towards Safe and Economic Laparoscopic Splenectomy. Kasr El Aini Journal of Surgery, 2009; 10(2): 17-33.

17. Fujioka S, Yoshida K, Okamoto T, Yanaga K. Staple-less Laparoscopic Splenectomy Using Harmonic Scalpel by 2-Step Sealing. Int Surg. 2013; 98: 385-387.

18. Amau T, Kojima K, Saka Y, Saiki Y, Otani T, Nishiwaki K, Aoki M, Ukai H. A Next - Generation Distribution Automation System Using IEC 61850 GOOSE and Section Switches with Sensors. Electrical Engineering in Japan, 2016; 195(2): 21-34.

19. Zhu FF, Poolton JM, Wilson MR, Hu Y, Maxwell JP, Masters RS. Implicit motor 
learning promotes neural efficiency during

laparoscopy. Surgical endoscopy, 2011;

25(9): 2950-5.

20. Hassan ME, Al Ali K. Massive splenomegaly in children: laparoscopic versus open splenectomy. JSLS: Journal of the Society of Laparoendoscopic Surgeons, 2014; 18(3): e2014.00245. 